\title{
Enhanced Efficiency Liquid Nitrogen Fertilizer Management for Corn Production
}

\author{
Tyler W. Steusloff, ${ }^{1}$ Gurbir Singh $\mathbb{D D}^{2,3}$ Kelly A. Nelson $\mathbb{D D}^{2}$, and Peter P. Motavalli ${ }^{1}$ \\ ${ }^{1}$ School of Natural Resources, University of Missouri, Columbia, Missouri 65211, USA \\ ${ }^{2}$ Lee Greenley Memorial Research Center, University of Missouri, Novelty, Missouri 63460, USA \\ ${ }^{3}$ Mississippi River Valley Alluvial Aquifer Water Center, Delta Research and Extension Center, Mississippi State University, \\ Stoneville, MS 38776, USA \\ Correspondence should be addressed to Gurbir Singh; singhgu@missouri.edu
}

Received 17 December 2018; Revised 21 June 2019; Accepted 2 July 2019; Published 5 August 2019

Academic Editor: Rodomiro Ortiz

Copyright (C) 2019 Tyler W. Steusloff et al. This is an open access article distributed under the Creative Commons Attribution License, which permits unrestricted use, distribution, and reproduction in any medium, provided the original work is properly cited.

\begin{abstract}
Claypan soils have a high potential for $\mathrm{N}$ loss, which can lower corn (Zea mays L.) yields. Field research was conducted from 2011 to 2013 in Northeast Missouri to determine corn yield, plant population, and grain quality response to $\mathrm{N}$ application timings (fall vs. spring) and five $\mathrm{N}$ sources/placements at two different $\mathrm{N}$ rates $\left(84\right.$ and $\left.168 \mathrm{~kg} \cdot \mathrm{N} \cdot \mathrm{ha} \mathrm{a}^{-1}\right)$ on a poorly drained claypan soil. The five $\mathrm{N}$ source/placement systems were no-till (NT)/surface broadcast urea ammonium nitrate (UAN) (Surface UAN) or strip-till (ST)/ deep banded UAN (deep UAN), NT/surface broadcast UAN plus Nitamin Nfusion (surface NF) or ST/deep banded UAN plus Nitamin Nfusion (deep NF), and ST/deep banded anhydrous ammonia (AA) (deep AA). The field trial was a split-plot randomized complete block design with four replications. Deep UAN with a fall $\mathrm{N}$ application produced the highest grain yield (8.12 to $9.12 \mathrm{Mg} \cdot \mathrm{ha}^{-1}$ ) at 84 and $168 \mathrm{~kg} \cdot \mathrm{N} \cdot \mathrm{ha}^{-1}$, but it was less effective with a spring application in 2011 . Fall deep AA produced the lowest grain yields $\left(5.97\right.$ and $6.8 \mathrm{Mg} \cdot \mathrm{ha}^{-1}$ ) in 2013 at 84 and $168 \mathrm{~kg} \cdot \mathrm{N} \cdot \mathrm{ha} \mathrm{a}^{-1}$ potentially due to wet soil conditions at the time of application. Warmer and wetter soil conditions during April-May of 2013 resulted in relatively higher grain yields compared to cooler and drier soil conditions in 2011 with all spring-applied N source/placement treatments. Extreme drought in the 2012 growing season resulted in poor corn growth. Farmers may need to consider fall $\mathrm{N}$ applications on claypan soils because spring $\mathrm{N}$ application might be riskier since corn grain yield was generally greater than or equal to spring-applied treatments.
\end{abstract}

\section{Introduction}

Nitrogen (N) management is a crucial and challenging component of sustainable corn production on poorly drained claypan soils. Claypan soils account for an area of 4 million hectares in the Midwest US in parts of Missouri, Kansas, and Illinois [1]. The low hydraulic conductivity of claypan soils makes these soils susceptible to waterlogged conditions following rainfall, which is an ideal environment for $\mathrm{N}$ loss through denitrification [2]. Saturated soil conditions may also lead to surface runoff resulting in $\mathrm{N}$ loss. The poor drainage properties of claypan soils can affect crop management decisions and reduce grain yields [3]. One proposed $\mathrm{N}$ management strategy for improving $\mathrm{N}$ utilization by crop plants and minimizing $\mathrm{N}$ loss is termed the
" $4 \mathrm{R}$ " strategy (i.e., right source, right rate, right time, and right place) [4]. For this strategy, $\mathrm{N}$ fertilizer timing, source, placement, or application rate are viewed as controllable factors that can improve corn yields in a poorly drained claypan soil. However, unpredictable weather conditions, including the distribution of precipitation and temperature, are uncontrollable factors that may cause the greatest impact on $\mathrm{N}$ use efficiency and yield.

In the Midwest US, a single preplant application of $\mathrm{N}$ fertilizer in the spring or soon after emergence is a commonly utilized $\mathrm{N}$ management practice to improve corn growth and development [5]. The consensus among researchers has been that the application of $\mathrm{N}$ fertilizer for corn should be applied nearest to the time $\mathrm{N}$ is needed by the crop and when $\mathrm{N}$ uptake is highest in order to achieve 
greater $\mathrm{N}$ use efficiency and to reduce $\mathrm{N}$ loss [6-8]. However, the time range for spring $\mathrm{N}$ application has become very narrow due to generally wetter spring conditions [9]. The risk of soil compaction and other challenges, such as greater $\mathrm{N}$ loss, with very wet soils are disadvantages for spring $\mathrm{N}$ application. In some instances, US corn growers prefer to apply $\mathrm{N}$ in the fall because labor is often more available, fertilizer prices are lower, and weather and soil conditions are more favorable than in the spring [10]. Fall $\mathrm{N}$ fertilizer application is riskier than spring application because the potential for $\mathrm{N}$ loss has increased as $\mathrm{N}$ was applied several months before the crop requirement [10]. A five-year study in Minnesota found 36\% or more N loss and an $8 \%$ reduction in corn grain yields with fall $\mathrm{N}$ application compared to a spring $\mathrm{N}$ application [11]. Randall et al. [12] found that a fall $\mathrm{N}$ application had higher $\mathrm{NO}_{3}-\mathrm{N}$ losses in subsurface drainage water compared to a spring $\mathrm{N}$ application. However, a study in Kansas reported no differences in corn grain yield between a fall and spring pre-plant $\mathrm{N}$ application [13]. Bundy [14] concluded that a fall $\mathrm{N}$ application was an acceptable option on clay textured soils if winter temperatures were below $10^{\circ} \mathrm{C}$ when nitrifying bacteria were less active. Since soils are generally below $10^{\circ} \mathrm{C}$ and lower rainfall occurs during fall on claypan soils of Northeast Missouri, corn response to $\mathrm{N}$ fertilizer application timings (fall vs. spring) needs to be evaluated.

In addition to $\mathrm{N}$ application timing, fertilizer $\mathrm{N}$ placement strategies may improve crop yields and $\mathrm{N}$ use efficiency in claypan soils. Many studies have shown lower grain yields and $\mathrm{N}$ uptake with a surface broadcast $\mathrm{N}$ application compared to incorporated band $\mathrm{N}$ placement $[6,15-17]$. Other studies did not observe any advantages of subsurface band placement of urea ammonium nitrate (UAN) over surface broadcast placement $[8,18]$. Placing $\mathrm{N}$ fertilizer below the soil surface can result in a higher concentration of nutrients within the root zone, which may increase $\mathrm{N}$ uptake and corn yields [19]. Surface applied N is commonly used with NT systems where high-residue levels can promote increased microbial activity near the soil surface, causing more potential for $\mathrm{N}$ loss [20]. Strip-till is a reduced tillage practice that allows for subsurface placement of $\mathrm{N}$ fertilizers within the tilled, planted row. Strip-till has similar benefits to NT systems, but ST can have an advantage because it increases internal drainage of the seedbed and initiates earlier warming of the soil in the spring due to less surface residue [21]. Benefits of NT depend on many factors including seasonal weather conditions and management practices such as $\mathrm{N}$ fertilizer timing, source, $\mathrm{N}$ placement, soil moisture content, soil temperature, and overall seedbed conditions in the spring $[22,23]$. However, surface application of N in NT or reduced tillage systems can be subject to $\mathrm{N}$ loss through denitrification and ammonia volatilization if $\mathrm{N}$ remains on the surface long enough and is exposed to the atmosphere $[24,25]$. In contrast, $\mathrm{N}$ loss can be reduced if $\mathrm{N}$ is placed below the soil surface [26].

Traditional $\mathrm{N}$ fertilizers used for corn production consist of dry, liquid, or gas-based sources. Increases in corn yield are largely dependent on the ability of the $\mathrm{N}$ source to provide inorganic, plant available forms of $\mathrm{N}\left(\mathrm{NH}_{4}{ }^{+}\right.$and
$\mathrm{NO}_{3}^{-}$) in sufficient concentrations in synchrony with plant $\mathrm{N}$ demand. Several slow- and controlled-release $\mathrm{N}$ fertilizers (SRF/CRF) have the potential to increase crop yield by improving the synchronization of plant $\mathrm{N}$ demand and $\mathrm{N}$ release [27-29]. The release rate of SRF/CRF can depend on soil properties, soil temperature, and microbial activity [8]. Previous studies have shown mixed (no change, increase, or decrease) results with use of SRF/CRFs compared to traditional fertilizer sources [6, 28-31]. Nitamin Nfusion (NF) is a new slow-release liquid enhanced efficiency $\mathrm{N}$ fertilizer product that contains $22 \% \mathrm{~N}$ of which $94 \%$ is slowly available in the form of methylene urea and triazone [32]. The product is completely water-soluble and can be blended with UAN, liquid urea, and other solutions at different ratios allowing for flexibility. Shapiro et al. [8] observed that surface broadcast UAN with NF and subsurface banded UAN with NF produced 0.21 to $0.59 \mathrm{Mg} \cdot \mathrm{ha}^{-1}$ higher grain yield than surface broadcast UAN without NF on a loamy sand soil at a site in Nebraska. However, there is a lack of research studies investigating the effects of $\mathrm{NF}$ and other $\mathrm{N}$ fertilizer solutions on corn production using a combination of different $\mathrm{N}$ timing, sources, and placements. The objective of this study was to determine the effects of two $\mathrm{N}$ application timings (fall vs. spring preplant) and five $\mathrm{N}$ sources/placements on corn yield, plant population, and grain quality in a poorly drained claypan soil.

\section{Materials and Methods}

2.1. Site Characterization. A three-year field experiment was conducted from 2011 to 2013 at the University of Missouri's Greenley Memorial Research Center $\left(40^{\circ} 1^{\prime} 17^{\prime \prime} \mathrm{N}\right.$, $92^{\circ} 11^{\prime} 24.9^{\prime \prime} \mathrm{W}$ ) on a poorly drained claypan soil (Putnam silt loam; fine smectitic, mesic Vertic Albaqualfs). The depth to the claypan at this research location was approximately $31 \mathrm{~cm}$ [33]. Experiments were conducted with corn on fields that were planted to soybean (Glycine max [L.] Merr.) the previous year in a dryland corn-soybean production system. Average monthly air temperature as well as daily and cumulative precipitation were obtained from a nearby automated weather station located at the Greenley Research Center near Novelty, MO.

2.2. Field Experiment and Layout. The field trial was a splitplot randomized complete block design with four replications. The main plot was $\mathrm{N}$ fertilizer application timing (fall and spring preplant), which was blocked and randomized within each replication. The sources/placements included $32 \%$ UAN NT/surface broadcast (surface UAN) or ST/deep banded (deep UAN), 32\% UAN plus Nitamin Nfusion (Koch Agronomic Services, Wichita, KS) NT/surface broadcast (surface NF) or ST/deep banded (Deep NF), and anhydrous ammonia deep banded (deep AA) only which were in a factorial arrangement with two application rates (84 and $168 \mathrm{~kg} \cdot \mathrm{N} \cdot \mathrm{ha}^{-1}$ ). Experimental plots were 3 by $23 \mathrm{~m}$, and the row spacing was $0.76 \mathrm{~m}$. Deep banded $\mathrm{N}$ fertilizer was applied to a depth of $20 \mathrm{~cm}$ with a custom-designed ST conservation C-jet unit (Advance, Missouri) [34]. Fall N application dates for 2011, 2012, and 2013 growing seasons were 11 Nov 2010, 
15 Nov 2011, and 11 Nov 2012, respectively. Spring preplant $\mathrm{N}$ application dates were $11 \mathrm{Apr}, 11 \mathrm{Apr}$, and 1 May for the 2011, 2012, and 2013 growing seasons, respectively. Corn hybrid, DeKalb 63-84, was planted on 12 Apr at 76,000 seeds.ha ${ }^{-1}$ in 2011, 11 Apr at 79,000 seeds.ha ${ }^{-1}$ in 2012, and 14 May at 81,500 seeds $\cdot \mathrm{ha}^{-1}$ in 2013. Maintenance fertilizer was only applied in 2012 at 17-35-100 (N-P-K) kg.ha ${ }^{-1}$ on 12 Apr. Corn was harvested on 13 Sep 2011, 23 Aug 2012, and 26 Sep 2013.

2.3. Data Collection. Stand counts for the two inner rows of each plot were recorded each year over a row length of $15.2 \mathrm{~m}$. Leaf chlorophyll measurements were recorded during the growing season on the most recent mature ear leaf halfway from the midrib of 10 plants.plot ${ }^{-1}$ at VT (tassel visible but before pollen shedding) [35] using a SPAD 502 Plus chlorophyll meter (Konica Minolta, Hong Kong) and were averaged for a whole plot value. Plant heights and stage of corn development were determined weekly until VT growth stage [35]. Corn test weight, grain moisture, and grain yields (Harvest Master, Logan, UT) were determined using a plot combine (Wintersteiger, Salt Lake City, UT) that harvested the two center rows in each plot which were $23 \mathrm{~m}$ in length. Grain yields were adjusted to $150 \mathrm{~g} \cdot \mathrm{kg}^{-1}$ moisture prior to analysis [3]. Grain samples were collected from each plot for determination of oil and protein concentration (Foss 1241 Infratec, Eden Prairie, MN).

2.4. Statistical Analysis. Data were analyzed for each rate ( 84 and $168 \mathrm{~kg} \cdot \mathrm{N} \cdot \mathrm{ha}^{-1}$ ) separately to detect differences between enhanced efficiency products compared to standard $\mathrm{N}$ treatments [36, 37]. Analysis of variance (ANOVA) was performed using the GLM procedure in the SAS v9.4 statistical program (SAS Institute, 2015). The dependent variables were year, source/placement, timing, and their interaction. Fisher's protected LSD at $P \leq 0.05$ was used to separate means and determine significant treatment effects.

\section{Results and Discussion}

3.1. Weather Conditions. Cumulative rainfall amounts for 2011, 2012, and 2013 growing seasons were 527, 239, and $468 \mathrm{~mm}$, respectively (Figure 1 ). The average 10 -year precipitation amount from 2000 to 2009 during the growing season (April through September) was $655 \mathrm{~mm}$ (University of Missouri Extension, 2018). The cumulative precipitation during the growing season of all three years was at least $25 \%$ lower than the 10 -year average. The growing season of 2012 experienced very little rainfall, and both the 2011 and 2013 growing seasons had 288 and $231 \mathrm{~mm}$ more cumulative rainfall than 2012, respectively. The average air temperature in 2011, 2012, and 2013 over the growing season was $20.9,22.0$, and $21.2^{\circ} \mathrm{C}$, respectively. The average 10 -year air temperature from 2000 to 2009 was $19.7^{\circ} \mathrm{C}$ over the growing season (University of Missouri Extension, 2018). All three years of this research were above the average air temperature. Average soil temperature over the growing season from 2000 to 2009 was $19.7^{\circ} \mathrm{C}$. Average soil temperature in 2011 was approximately the same $\left(19.7^{\circ} \mathrm{C}\right)$ as the prior ten season average, while 2012 was slightly higher $\left(20.8^{\circ} \mathrm{C}\right)$ and 2013 slightly lower $\left(19.3^{\circ} \mathrm{C}\right)$ (Table 1$)$. Soil temperatures were mostly below $10^{\circ} \mathrm{C}$ from November to March in the 2010-2011 and 2012-2013 study years which is ideal to minimize $\mathrm{N}$ loss with fall $\mathrm{N}$ application [38].

An assessment of total rainfall, average air temperature, and soil temperature during portions (e.g., fall and spring) of each year's study period may account for differences in the observed corn response due to $\mathrm{N}$ management systems among study years. Over the period of fall $\mathrm{N}$ application to planting in 2012-2013, total precipitation was 288 and $75 \mathrm{~mm}$ greater than in 2010-2011 and 2011-2012, respectively; however, cumulative rainfall in the 2012-2013 period was greater because of a relatively wetter April. Planting was delayed until 1 May in 2013 because spring precipitation was 99\% higher in April 2013 compared to the 10-year average. Cumulative rainfall in 2013 was 126 and $105 \mathrm{~mm}$ higher in the first four weeks after planting than in 2011 and 2012, respectively. Cumulative rainfall observed in June was 135 and $100 \mathrm{~mm}$ higher in 2011 compared to 2012 and 2013, respectively. In 2011 and 2013, July had similar rainfall (50 and $48 \mathrm{~mm})$ and was greater than July $2012(19 \mathrm{~mm})$ cumulative rainfall. Rainfall events in August 2013 and August 2011 had $48 \mathrm{~mm}$ more cumulative rainfall than August 2012.

3.2. Early-Season Corn Plant Height. Corn plant heights measured on 1 June and 30 June had significant $(P \leq 0.05)$ differences among $\mathrm{N}$ source placements at $84 \mathrm{~kg} \cdot \mathrm{N} \cdot \mathrm{ha}^{-1}$ (Table 2). Surface UAN and surface NF had significantly greater corn plant heights on 1 June than deep UAN and deep NF at $84 \mathrm{~kg} \cdot \mathrm{N} \cdot \mathrm{ha}^{-1}$ (Figure 2(a)). Similarly, surface UAN and surface NF had greater corn plant heights on 30 June than deep UAN and NF treatments at $84 \cdot \mathrm{kg} \cdot \mathrm{N} \cdot \mathrm{ha}^{-1}$ (Figure 2(a)). Corn plant height measured on 15 June was significantly $(P<0.05)$ different among $\mathrm{N}$ timing at $84 \mathrm{~kg} \cdot \mathrm{N} \cdot \mathrm{ha}^{-1}$ (Table 2). Fall $\mathrm{N}$ application had a mean corn plant height that was $2.6 \mathrm{~cm}$ higher than spring $\mathrm{N}$ application at $84 \mathrm{~kg} \cdot \mathrm{N} \cdot \mathrm{ha}^{-1}$ on 15 June (Figure 3(a)). This suggests that corn plant height can be an indicator for slightly greater growth since fall $\mathrm{N}$ application had taller plants $\mathrm{ha}^{-1}$ at $84 \mathrm{~kg} \cdot \mathrm{N} \cdot \mathrm{ha}^{-1}$.

At $168 \mathrm{~kg} \cdot \mathrm{N} \cdot \mathrm{ha}^{-1}$, corn plant height measured on 27 May, 8 June, and 15 June was significantly $(P \leq 0.05)$ different due to $\mathrm{N}$ sources/placements (Table 2). Surface NF $(14.2 \mathrm{~cm})$ was 0.4 to $1.6 \mathrm{~cm}$ taller than deep UAN, deep NF, and deep AA treatments on 27 May (Figure 2(b)). Surface UAN also had significantly greater corn plant heights than all deep banded treatments on 8 June and 15 June. Lower early-season plant heights for ST/deep banded treatments suggested that plant emergence and growth were delayed. These results were counter to results from a study in Indiana that found lower early-season corn plant heights and delayed maturity in treatments with NT compared to conventional tillage [39]. Delayed plant emergence may result from a variety of factors including cooler soil temperatures, higher soil moisture, and high soil residue cover [40], which is typically associated with NT systems. The NT systems may 


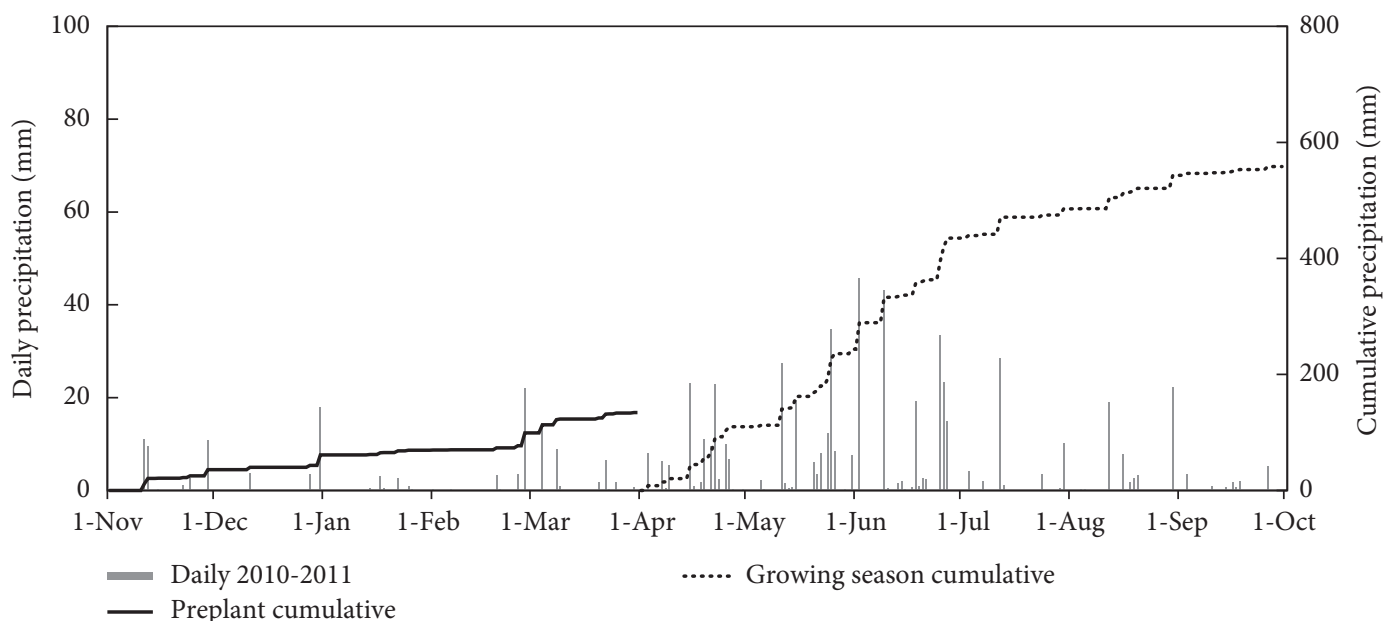

(a)

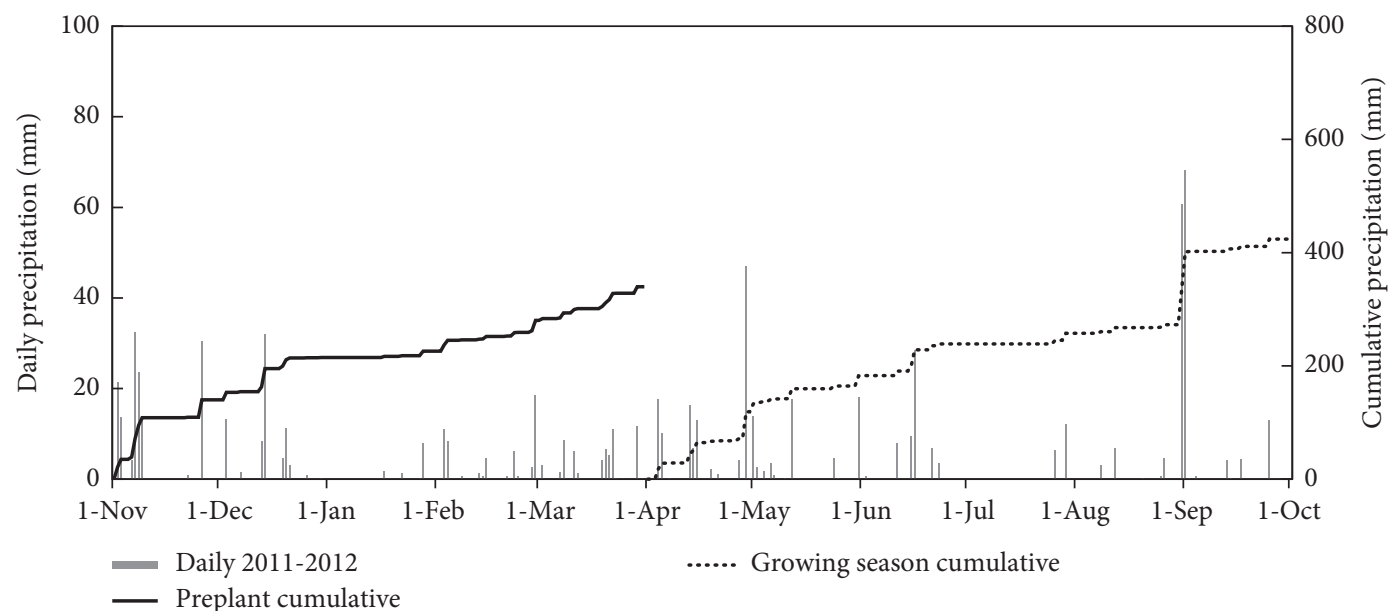

(b)

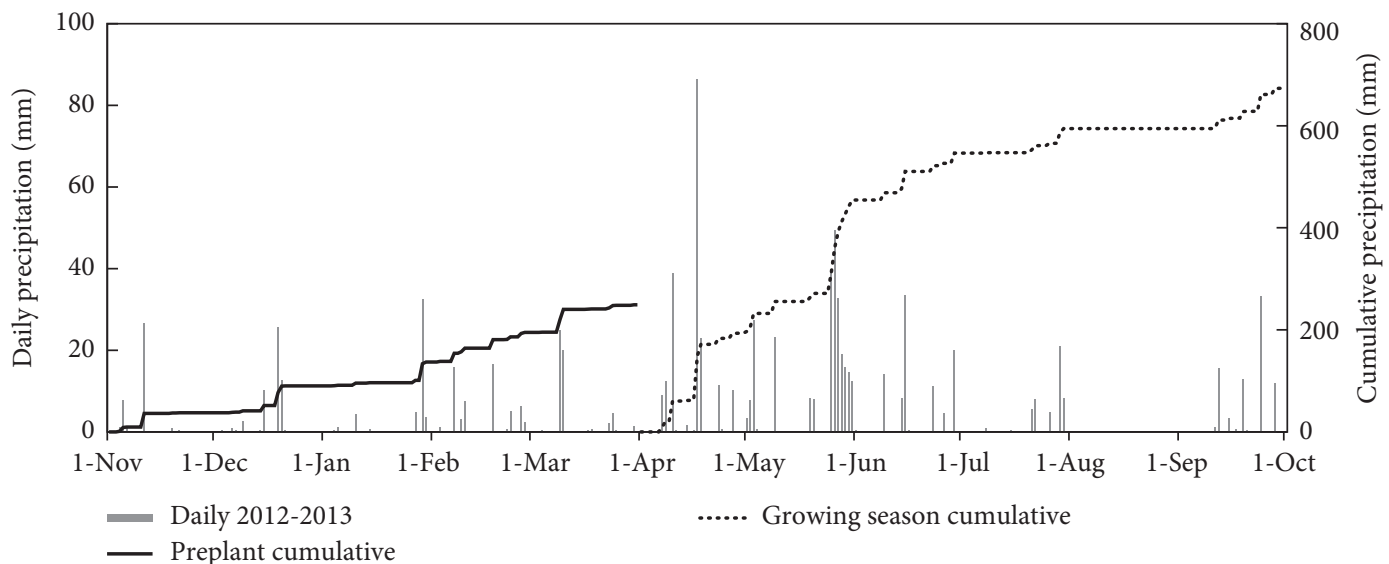

(c)

FIGURE 1: Daily and cumulative precipitation history for (a) 2010-2011, (b) 2011-2012, and (c) 2012-2013.

have cooler, wetter seedbed conditions [21] and can have reduced corn plant heights when combined with a surface $\mathrm{N}$ application due to greater $\mathrm{N}$ loss [20].

Corn plant height had significant $(P \leq 0.05)$ differences at $168 \mathrm{~kg} \cdot \mathrm{N} \cdot \mathrm{ha}^{-1}$ among $\mathrm{N}$ timings (fall or spring) on 27 May, 1 June, 15 June, and 22 June (Table 2). Fall $\mathrm{N}$ applications had taller plants than spring $\mathrm{N}$ treatments (Figure 3(b)). This suggests that greater early-season plant heights with fall $\mathrm{N}$ application may not always indicate greater plant heights at later corn growth stages when $\mathrm{N}$ was applied at a higher rate $\left(168 \mathrm{~kg} \cdot \mathrm{N} \cdot \mathrm{ha}^{-1}\right)$. Fall $\mathrm{N}$ application generally has larger amounts of $\mathrm{NO}_{3}-\mathrm{N}$ available in the spring [7], which suggests 
TABLE 1: Average monthly soil temperature obtained from a nearby automated weather station located at the Greenley Research Center near Novelty, MO, at the $6 \mathrm{~cm}$ depth with soybean residue from the time of fall $\mathrm{N}$ application to corn harvest in each season.

\begin{tabular}{lccc}
\hline Month & \multicolumn{2}{c}{ Soil temperature $\left({ }^{\circ} \mathrm{C}\right)$} \\
$2011-2012$ & $2012-2013$ \\
\hline Nov & $2010-2011$ & 7.7 & 6.7 \\
Dec & 7.3 & 3.5 & 3.9 \\
Jan & 0.8 & 1.4 & 0.9 \\
Feb & -0.3 & 2.5 & 0.9 \\
Mar & 0.3 & 10.2 & 2.1 \\
Apr & 5.5 & 13 & 9 \\
May & 10.7 & 19.4 & 16 \\
Jun & 16.2 & 22.6 & 21.2 \\
Jul & 21.7 & 27 & 23.5 \\
Aug & 25.8 & 23.1 & 28.3 \\
Sep & 24.6 & 19.2 & 22 \\
\hline
\end{tabular}

TABle 2: The ANOVA table for corn plant heights at 84 and $168 \mathrm{~kg} \cdot \mathrm{N} \cdot \mathrm{ha}{ }^{-1}$.

\begin{tabular}{|c|c|c|c|c|c|c|c|c|}
\hline Source of variation & $d f$ & $\begin{array}{c}27 \text { May } \\
\operatorname{Pr}>\text { F }\end{array}$ & $\begin{array}{l}1 \text { June } \\
\operatorname{Pr}>\mathrm{F}\end{array}$ & $\begin{array}{l}8 \text { June } \\
\operatorname{Pr}>\mathrm{F}\end{array}$ & $\begin{array}{c}15 \text { June } \\
\operatorname{Pr}>\mathrm{F}\end{array}$ & $\begin{array}{c}22 \text { June } \\
\operatorname{Pr}>\mathrm{F}\end{array}$ & $\begin{array}{c}30 \text { June } \\
\operatorname{Pr}>\mathrm{F}\end{array}$ & $\begin{array}{l}6 \text { July } \\
\operatorname{Pr}>\text { F }\end{array}$ \\
\hline \multicolumn{9}{|c|}{$\mathrm{N}$ application rate $=84 \mathrm{~kg} \cdot \mathrm{N} \cdot \mathrm{ha}^{-1}$} \\
\hline Year & 2 & $<0.0001^{\ddagger}$ & $<0.0001$ & $<0.0001$ & $<0.0001$ & $<0.0001$ & $<0.0001$ & $<0.0001$ \\
\hline Timing & 1 & 0.0909 & 0.2542 & 0.0502 & 0.0080 & 0.1304 & 0.4199 & 0.1464 \\
\hline Year $\times$ timing & 2 & 0.1699 & 0.1770 & 0.0843 & 0.0021 & 0.0250 & 0.0054 & $<0.0001$ \\
\hline SorcPlac ${ }^{\dagger}$ & 4 & 0.0878 & 0.0060 & 0.1925 & 0.1535 & 0.9851 & 0.0337 & 0.2099 \\
\hline Year $\times$ SorcPlac & 8 & 0.5414 & 0.0543 & 0.1082 & 0.0937 & 0.7844 & 0.0257 & 0.1137 \\
\hline SorcPlac $\times$ timing & 4 & 0.2358 & 0.1852 & 0.3019 & 0.2454 & 0.1515 & 0.2543 & 0.0973 \\
\hline Year $\times$ SorcPlac $\times$ timing & 8 & 0.8873 & 0.1855 & 0.5453 & 0.6266 & 0.9517 & 0.9865 & 0.4783 \\
\hline \multicolumn{9}{|c|}{$\mathrm{N}$ application rate $=168 \mathrm{~kg} \cdot \mathrm{N} \cdot \mathrm{ha}^{-1}$} \\
\hline Year & 2 & $<0.0001^{\ddagger}$ & $<0.0001$ & $<0.0001$ & $<0.0001$ & $<0.0001$ & $<0.0001$ & $<0.0001$ \\
\hline Timing & 1 & 0.0111 & 0.0001 & 0.3070 & 0.0071 & 0.0224 & 0.1618 & 0.1267 \\
\hline Year $\times$ timing & 2 & 0.0010 & $<0.0001$ & 0.1474 & 0.0030 & 0.0198 & 0.0207 & $<0.0001$ \\
\hline SorcPlac $^{\dagger}$ & 4 & 0.0001 & 0.0909 & 0.0113 & 0.0345 & 0.3286 & 0.1776 & 0.1033 \\
\hline Year $\times$ SorcPlac & 8 & 0.0473 & 0.0759 & 0.0001 & 0.1302 & 0.3409 & 0.2188 & 0.0950 \\
\hline SorcPlac $\times$ timing & 4 & 0.4751 & 0.0007 & 0.0361 & 0.1839 & 0.3107 & 0.1450 & 0.2826 \\
\hline Year $\times$ SorcPlac $\times$ timing & 8 & 0.3364 & 0.0495 & 0.2730 & 0.5143 & 0.7584 & 0.4955 & 0.7226 \\
\hline
\end{tabular}

${ }^{\dagger}$ Abbreviations: SorcPlac, source/placement. ${ }^{*}$ Numbers in bold represent Fisher's protected least significant difference at $P \leq 0.05$.

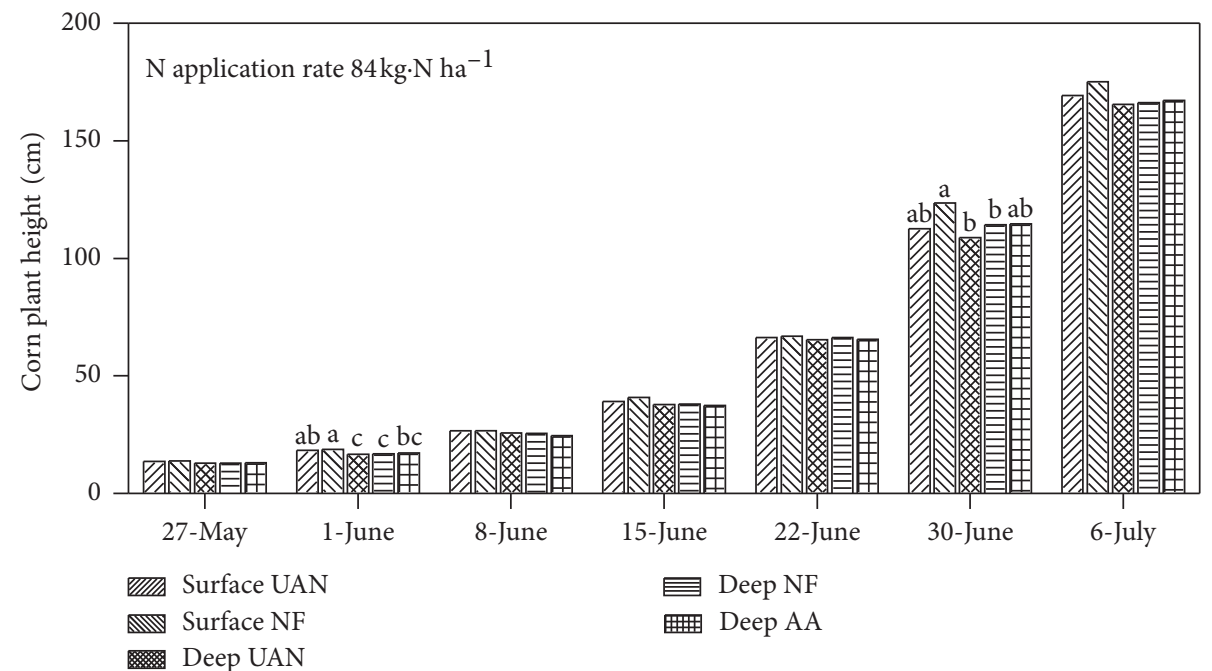

(a)

Figure 2: Continued. 


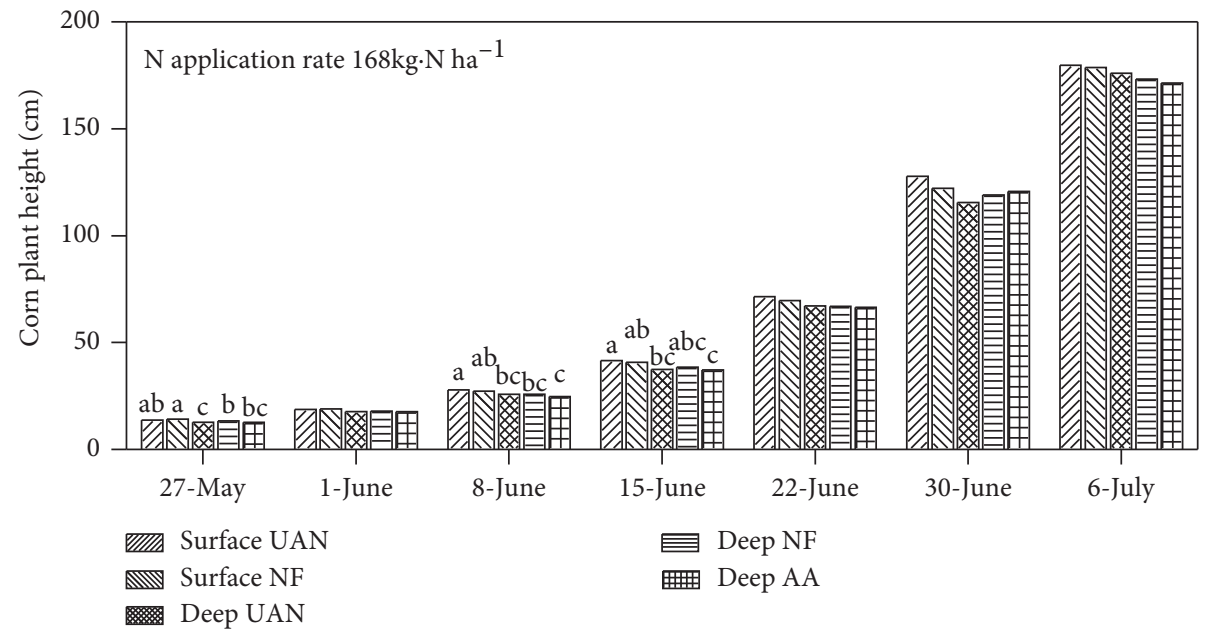

(b)

Figure 2: Corn plant heights due to $\mathrm{N}$ source placements averaged over $\mathrm{N}$ timing and years. Plant heights were measured on selected dates before VT at (a) 84 and (b) $168 \mathrm{~kg} \cdot \mathrm{N} \cdot \mathrm{ha}^{-1}$. Letters over bars indicate differences among treatments within a given measurement date using Fisher's Protected LSD $(P \leq 0.05)$.

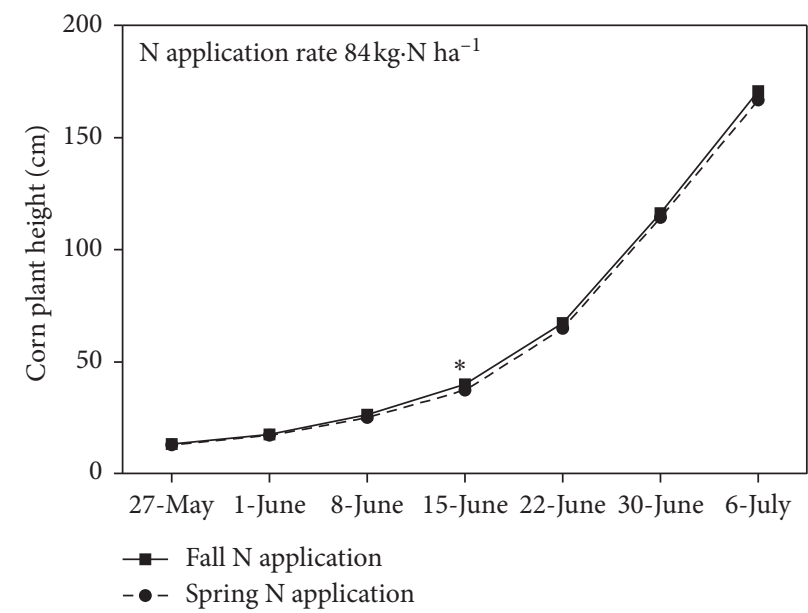

(a)

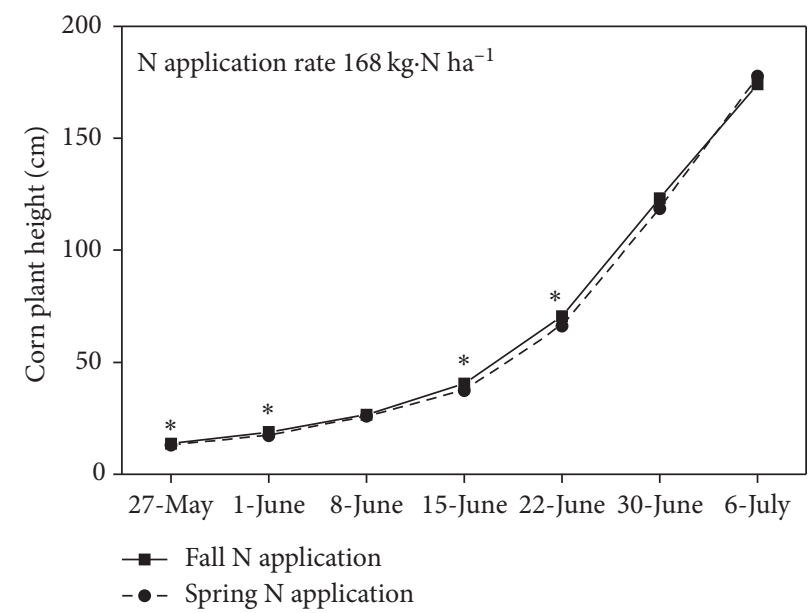

(b)

Figure 3: Corn plant heights due to $\mathrm{N}$ timing averaged over $\mathrm{N}$ source placements and years. Plant heights were measured on selected dates before VT at (a) 84 and (b) $168 \mathrm{~kg} \cdot \mathrm{N} \cdot \mathrm{ha}^{-1}$. Asterisks over lines indicate differences among treatments within a given measurement date using Fisher's Protected LSD $(P \leq 0.05)$.

that corn readily utilized $\mathrm{N}$ at the early growth stages in the spring, which contributed to the early growth and plant vigor.

3.3. Ear Leaf Chlorophyll Meter Readings. A three-way interaction between source/placement, timing, and year was found at $84 \mathrm{~kg} \cdot \mathrm{N} \cdot \mathrm{ha}^{-1}$ for corn chlorophyll meter readings (Table 3). Chlorophyll meter readings were generally 4.7 SPAD units higher in 2011 and 2012 among all N sources/ placements compared to 2013 regardless of $\mathrm{N}$ application timing (Table 4). Deep UAN, deep NF, and deep AA resulted in at least 2.5 SPAD unit higher readings than surface UAN and surface NF in 2011 and 2012 regardless of $\mathrm{N}$ application timing. Chlorophyll meter readings for fall and spring-applied deep NF were $8 \%$ higher compared to surface NF in 2011 and 2012. Applying $\mathrm{N}$ in the fall or spring had no significant differences in chlorophyll meter readings within $\mathrm{N}$ sources/ placements in 2011 and 2012. In 2013, chlorophyll meter readings indicated that plants were $22 \%$ greener with springapplied deep AA compared to fall-applied deep AA. Conversely, chlorophyll meter readings were $11 \%$ greater with fall-applied deep NF compared to spring-applied deep NF.

Nitrogen applied at $168 \mathrm{~kg} \cdot \mathrm{ha}^{-1}$ had chlorophyll meter readings values with a significant interaction between year and $\mathrm{N}$ source placement $(P \leq 0.05)$ (Table 3 ). Similar to $\mathrm{N}$ applied at $84 \mathrm{~kg} \cdot \mathrm{ha}^{-1}$, chlorophyll meter readings in 2011 and 2012 were highest (50.7 to 57.3 SPAD units) among all $\mathrm{N}$ sources/placements at $168 \mathrm{~kg} \cdot \mathrm{N} \cdot \mathrm{ha}^{-1}$ compared to that in 
TABLE 3: The ANOVA table for chlorophyll meter readings, plant population, yield, moisture, test weight, starch, protein, and oil concentration for nitrogen treatments at 84 and $168 \mathrm{~kg} \cdot \mathrm{N} \cdot \mathrm{ha}{ }^{-1}$.

\begin{tabular}{|c|c|c|c|c|c|c|c|c|}
\hline Source of variation & $d f$ & $\begin{array}{l}\text { Chlorophyll meter readings } \\
\qquad \operatorname{Pr}>\mathrm{F}\end{array}$ & $\begin{array}{l}\text { Population } \\
\qquad \operatorname{Pr}>F\end{array}$ & $\begin{array}{l}\text { Yield } \\
\text { Pr }>\text { F }\end{array}$ & $\begin{array}{l}\text { Moisture } \\
\operatorname{Pr}>\mathrm{F}\end{array}$ & $\begin{array}{l}\text { Test weight } \\
\operatorname{Pr}>\mathrm{F}\end{array}$ & $\begin{array}{l}\text { Protein } \\
\operatorname{Pr}>\mathrm{F}\end{array}$ & $\begin{array}{c}\text { Oil } \\
\text { Pr }>\text { F }\end{array}$ \\
\hline \multicolumn{9}{|c|}{$\mathrm{N}$ application rate $=84 \mathrm{~kg} \cdot \mathrm{N} \cdot \mathrm{ha}^{-1}$} \\
\hline Year $^{\dagger}$ & 2 & $<0.0001^{*}$ & $<0.0001$ & $<0.0001$ & 0.0604 & 0.3102 & $<0.0001$ & $<0.0001$ \\
\hline Timing & 1 & 0.5156 & $<0.0001$ & 0.0005 & 0.1431 & 0.4947 & 0.8297 & 0.676 \\
\hline Year $\times$ timing & 2 & 0.748 & $<0.0001$ & $<0.0001$ & 0.0053 & 0.4133 & 0.0312 & 0.6729 \\
\hline SorcPlac $^{\dagger}$ & 4 & $<0.0001$ & $<0.0001$ & 0.0056 & 0.0023 & 0.4348 & $<0.0001$ & 0.3492 \\
\hline Year $\times$ SorcPlac & 8 & 0.0053 & $<0.0001$ & 0.445 & 0.0031 & 0.228 & $<0.0001$ & 0.9257 \\
\hline SorcPlac $\times$ timing & 4 & 0.6319 & 0.0187 & 0.0022 & $0.0018^{\dagger}$ & 0.8582 & 0.325 & 0.5522 \\
\hline Year $\times$ SorcPlac $\times$ timing & 8 & 0.0285 & 0.0698 & 0.0046 & 0.4078 & 0.2404 & 0.1042 & 0.2024 \\
\hline \multicolumn{9}{|c|}{$\mathrm{N}$ application rate $=168 \mathrm{~kg} \cdot \mathrm{N} \cdot \mathrm{ha}^{-1}$} \\
\hline Year & 2 & $<0.0001^{\ddagger}$ & $<0.0001$ & $<0.0001$ & $<0.0001$ & 0.0311 & $<0.0001$ & $<0.0001$ \\
\hline Timing & 1 & 0.3242 & $<0.0001$ & 0.0009 & 0.4513 & 0.1823 & 0.3167 & 0.0356 \\
\hline Year $\times$ timing & 2 & 0.1093 & $<0.0001$ & $<0.0001$ & 0.1353 & 0.5255 & 0.1334 & 0.2038 \\
\hline SorcPlac $^{\dagger}$ & 4 & 0.0028 & $<0.0001$ & 0.1721 & $<0.0001$ & 0.2391 & $<0.0001$ & 0.0132 \\
\hline Year $\times$ SorcPlac & 8 & 0.0054 & $<0.0001$ & 0.0327 & 0.1372 & 0.3354 & $<0.0001$ & 0.1423 \\
\hline SorcPlac $\times$ timing & 4 & 0.0809 & 0.0029 & 0.0873 & 0.3683 & 0.4364 & 0.1826 & 0.2722 \\
\hline Year $\times$ SorcPlac $\times$ timing & 8 & 0.4748 & 0.1815 & 0.0231 & 0.3078 & 0.4866 & 0.1145 & 0.6455 \\
\hline
\end{tabular}

${ }^{\dagger}$ Abbreviations: SorcPlac, source/placement. ${ }^{*}$ Numbers in bold represent Fisher's protected least significant difference at $P \leq 0.05$.

TABLE 4: Corn SPAD, plant population, and grain yield response to nitrogen sources/placements at $84 \mathrm{~kg} \cdot \mathrm{N} \cdot \mathrm{ha}{ }^{-1}$. Data were combined over factors or years in the absence of a significant interaction. Surface treatments were no-tillage with surface applied nitrogen and deep treatments were strip tillage with deep banded nitrogen application.

\begin{tabular}{|c|c|c|c|c|c|c|c|c|c|c|c|c|c|c|}
\hline \multirow{3}{*}{$\mathrm{N}$ source placement } & \multicolumn{6}{|c|}{ Chlorophyll meter readings } & \multirow{2}{*}{\multicolumn{2}{|c|}{ Population }} & \multicolumn{6}{|c|}{ Grain yield } \\
\hline & \multicolumn{2}{|c|}{2011} & \multicolumn{2}{|c|}{2012} & \multicolumn{2}{|c|}{2013} & & & \multicolumn{2}{|c|}{2011} & \multicolumn{2}{|c|}{2012} & \multicolumn{2}{|c|}{2013} \\
\hline & $\mathrm{F}$ & S & $\mathrm{F}$ & S & $\mathrm{F}$ & S & $\mathrm{F}$ & S & $\mathrm{F}$ & S & $\mathrm{F}$ & S & $\mathrm{F}$ & S \\
\hline & \multicolumn{6}{|c|}{ SPAD units } & \multicolumn{2}{|c|}{ No. ha ${ }^{-1}$} & \multicolumn{6}{|c|}{$\mathrm{Mg} \cdot \mathrm{ha}^{-1}$} \\
\hline Surface $\mathrm{UAN}^{\dagger}$ & 48.2 & 44.6 & 47.9 & 48.8 & 39.2 & 39.3 & 70,000 & 67,800 & 6.91 & 5.98 & 2.14 & 2.30 & 6.53 & 7.23 \\
\hline Deep UAN & 54.4 & 53.4 & 51.3 & 53.7 & 44.0 & 44.0 & 66,750 & 56,100 & 8.12 & 3.91 & 1.73 & 2.11 & 7.62 & 7.53 \\
\hline Surface NF & 46.8 & 47.8 & 48.6 & 47.8 & 41.7 & 42.1 & 70,400 & 70,050 & 6.85 & 5.70 & 2.02 & 2.50 & 6.82 & 7.75 \\
\hline Deep NF & 52.1 & 55.3 & 52.4 & 53.4 & 41.8 & 37.8 & 68,500 & 59,150 & 7.01 & 4.72 & 1.33 & 1.76 & 7.17 & 6.70 \\
\hline Deep AA & 55.7 & 55.2 & 53.8 & 52.5 & 37.5 & 45.8 & 64,200 & 60,100 & 6.70 & 4.41 & 1.60 & 1.70 & 5.97 & 7.58 \\
\hline LSD $(P \leq 0.05)^{\ddagger}$ & \multicolumn{6}{|c|}{4.7} & \multicolumn{2}{|c|}{8,700} & \multicolumn{6}{|c|}{0.95} \\
\hline
\end{tabular}

${ }^{\dagger}$ Abbreviations: AA, anhydrous ammonia; F, fall; NF, Nitamin Nfusion; S, spring; UAN, $32 \%$ urea ammonium nitrate. ${ }^{\text {*Fisher's protected least significant }}$ difference at $P \leq 0.05$.

2013 (Table 5). There were no significant differences in SPAD readings in 2011 compared to that in 2012 among $\mathrm{N}$ sources/placements. Deep UAN, deep NF, and deep AA resulted in 0.3 to 6.6 higher SPAD units than surface UAN and surface NF in 2011 and 2012. Although not significantly different in 2012, deep NF resulted in the highest SPAD readings in 2011,2012 , and 2013 by $1.1,1.3$, and 1.9 SPAD units, respectively.

Chlorophyll meter readings may have been more affected by fall-applied treatments due to the distribution and amount of precipitation during the nongrowing season months of November through March. For example, 2013 was relatively wet early in the growing season and these conditions may have affected spring-applied deep AA at $68 \mathrm{~kg} \cdot \mathrm{N} \cdot \mathrm{ha}^{-1}$ compared to fall-applied. Furthermore, cumulative precipitation in the months after fall $\mathrm{N}$ application (November 2012) to planting was the wettest among the three years and potentially caused increased $\mathrm{N}$ loss due to denitrification [7]. Nevertheless, chlorophyll meter readings in 2011 and 2012 were higher in all deep banded treatments.
Previous studies indicated that deep banding $\mathrm{N}$ lowered the rates of microbial $\mathrm{N}$ transformations over surface application, which limited the amount of $\mathrm{N}$ that is lost through denitrification and volatilization $[41,42]$.

3.4. Plant Population. Plant populations had an interaction between $\mathrm{N}$ source/placement and $\mathrm{N}$ timing $(P \leq 0.05)$ at $84 \mathrm{~kg} \cdot \mathrm{N} \cdot \mathrm{ha}^{-1}$ (Table 3$)$. Plant populations for fall-applied $\mathrm{N}$ at $84 \mathrm{~kg} \cdot \mathrm{N} \cdot \mathrm{ha}^{-1}$ in the deep UAN and deep NF treatments were 16 and $14 \%$ higher than spring-applied $\mathrm{N}$, respectively (Table 4). When $\mathrm{N}$ was applied at $168 \mathrm{~kg} \cdot \mathrm{N} \cdot \mathrm{ha}^{-1}$ in the fall, deep NF had plant populations that were 15,650 plants. $\mathrm{ha}^{-1}$ greater than spring-applied $\mathrm{N}$ (Table 5). Although there were no significant differences between $\mathrm{N}$ timings among the other $\mathrm{N}$ source/placement treatments, fall-applied $\mathrm{N}$ at $84 \mathrm{~kg} \cdot \mathrm{N} \cdot \mathrm{ha}^{-1}$ had higher plant populations (350 to 4,100 plants $\cdot \mathrm{ha}^{-1}$ ) for surface UAN, surface NF, and deep AA treatments compared to spring $\mathrm{N}$ application. 
TABLE 5: Chlorophyll meter readings, plant population, grain yield, moisture, protein, and oil concentration response to nitrogen sources/ placements at $168 \mathrm{~kg} \cdot \mathrm{N} \cdot \mathrm{ha}^{-1}$. Data were combined over factors or years in the absence of a significant interaction. Surface treatments were no-tillage with surface applied nitrogen and deep treatments were strip tillage with deep banded nitrogen application.

\begin{tabular}{|c|c|c|c|c|c|c|c|c|c|c|c|c|c|c|c|c|}
\hline \multirow{4}{*}{$\mathrm{N}$ source placement } & \multirow{2}{*}{\multicolumn{3}{|c|}{$\begin{array}{l}\text { Chlorophyll meter } \\
\text { readings }\end{array}$}} & \multirow{3}{*}{\multicolumn{2}{|c|}{$\begin{array}{l}\text { Corn plant } \\
\text { population }\end{array}$}} & \multicolumn{11}{|c|}{ Corn grain parameters } \\
\hline & & & & & & \multirow{2}{*}{\multicolumn{2}{|c|}{2011}} & \multicolumn{2}{|c|}{ Yield } & & & \multirow[t]{2}{*}{ Moisture } & \multicolumn{3}{|c|}{ Protein } & \multirow[t]{3}{*}{ Oil } \\
\hline & \multirow[t]{2}{*}{2011} & \multirow[t]{2}{*}{2012} & \multirow[t]{2}{*}{2013} & & & & & \multicolumn{2}{|c|}{2012} & \multicolumn{2}{|c|}{2013} & & \multirow[t]{2}{*}{2011} & \multirow[t]{2}{*}{2012} & \multirow[t]{2}{*}{2013} & \\
\hline & & & & $\mathrm{F}$ & S & $\mathrm{F}$ & S & $\mathrm{F}$ & S & $\mathrm{F}$ & S & & & & & \\
\hline & \multicolumn{3}{|c|}{ SPAD units } & \multicolumn{2}{|c|}{ No. ha ${ }^{-1}$} & \multicolumn{6}{|c|}{${\mathrm{Mg} \cdot \mathrm{ha}^{-1}}$} & \multicolumn{5}{|c|}{$\mathrm{g} \cdot \mathrm{kg}^{-1}$} \\
\hline Surface $\mathrm{UAN}^{\dagger}$ & 55.6 & 52.8 & 45.9 & 71,100 & 70,300 & 8.94 & 8.11 & 1.41 & 1.75 & 8.08 & 8.16 & $181 b$ & 77.8 & 103.8 & 75.9 & 32.7 \\
\hline Deep UAN & 57.3 & 56.9 & 50.7 & 66,700 & 57,000 & 9.12 & 5.52 & 1.27 & 1.35 & 8.46 & 8.60 & $190 \mathrm{~b}$ & 92.5 & 104.5 & 88.4 & 31.1 \\
\hline Surface NF & 50.7 & 53.6 & 48.8 & 73,050 & 70,450 & 8.56 & 7.38 & 1.63 & 1.79 & 8.01 & 8.68 & $175 b$ & 77.5 & 103.9 & 84.4 & 31.4 \\
\hline Deep NF & 56.2 & 55.6 & 44.7 & 69,900 & 54,250 & 8.47 & 6.06 & 1.30 & 1.78 & 8.42 & 8.36 & $207 a$ & 93.8 & 104.8 & 83.1 & 32.6 \\
\hline Deep AA & 55.9 & 55.4 & 43.8 & 64,000 & 58,650 & 9.06 & 6.36 & 1.44 & 1.71 & 6.80 & 8.50 & $215 a$ & 92.0 & 104.3 & 79.9 & 33.1 \\
\hline $\operatorname{LSD}(P \leq 0.05)^{\ddagger}$ & \multicolumn{3}{|c|}{5.2} & \multicolumn{2}{|c|}{9,800} & \multicolumn{6}{|c|}{1.03} & 16 & & 6.9 & & 1.3 \\
\hline
\end{tabular}

${ }^{\dagger}$ Abbreviations: AA, anhydrous ammonia; F, fall; NF, Nitamin Nfusion; S, spring; UAN, 32\% urea ammonium nitrate. ${ }^{*}$ Fisher's protected least significant difference at $P \leq 0.05$.

There was also an interaction between $\mathrm{N}$ source/placement and $\mathrm{N}$ timing $(P<0.05)$ at $168 \mathrm{~kg} \cdot \mathrm{N} \cdot \mathrm{ha}^{-1}$ (Table 3$)$. Plant populations were 800 to 9,700 plants.ha ${ }^{-1}$ higher with fall-applied $\mathrm{N}$ at $168 \mathrm{~kg} \cdot \mathrm{ha}^{-1}$ in treatments other than deep $\mathrm{NF}$ compared to spring-applied $\mathrm{N}$ (Table 5). Both $\mathrm{N}$ rates increased plant population for deep NF when $\mathrm{N}$ was fallapplied. Deep NF was applied with ST which may have improved seedbed conditions more effectively compared to those of NT treatments [21, 43]. Hendrix et al. [23] found that NT on a silt loam soil had $37 \%$ lower plant populations than with ST, potentially due to NT having prolonged saturated soil conditions in the row. Extreme rainfall following spring ST may have caused a reduction in plant populations compared to fall ST which may have been a firmer seedbed.

3.5. Corn Grain Yield. Corn grain yields were observed to have a significant three-way interaction $(P \leq 0.05)$ between the timing of $\mathrm{N}$ application, source/placement, and year at $84 \mathrm{~kg} \cdot \mathrm{N} \cdot \mathrm{ha}^{-1}$ (Table 3). In 2011, deep UAN had 1.11 to $1.42 \mathrm{Mg} \cdot \mathrm{ha}^{-1}$ greater corn grain yield than all of the $\mathrm{N}$ sources/placements with fall $\mathrm{N}$ application (Table 4). Spring application of deep UAN had $4.21 \mathrm{Mg} \cdot \mathrm{ha}^{-1}$ lower corn grain yields in 2011 compared to fall-applied deep UAN. Strip tillage for corn in the fall can increase grain yield due to more favorable and drier soil conditions, settling of soil in a tilled row during winter, and warmer and drier seedbed conditions by spring [21]. Fall $\mathrm{N}$ application for all $\mathrm{N}$ treatments resulted in 0.93 to $4.21 \mathrm{Mg} \cdot \mathrm{ha}^{-1}$ greater corn grain yields in 2011 compared to spring-applied N. Regardless of N timing, the severe drought in 2012 (NAOO, 2013) resulted in average corn grain yields of $1.9 \mathrm{Mg} \cdot \mathrm{ha}^{-1}$ which were $4.1 \mathrm{Mg} \cdot \mathrm{ha}^{-1}$ lower compared to those in 2011 and 2013. Corn grain yields were similar among sources/placements and timings in 2012. In 2013, deep UAN and deep NF showed no significant differences in corn grain yield when $\mathrm{N}$ was applied in the fall or spring. Spring application of surface NF in 2013 had the highest corn grain yield $\left(7.75 \mathrm{Mg} \cdot \mathrm{ha}^{-1}\right)$ and produced at least $0.17 \mathrm{Mg} \cdot \mathrm{ha}^{-1}$ more grain yield among the spring-applied $\mathrm{N}$ treatments.
Corn grain yield in 2013 with deep AA was $1.61{\mathrm{Mg} \cdot \mathrm{ha}^{-1}}^{-1}$ (27\%) greater with spring $\mathrm{N}$ application compared to fall application (Table 4). Kyveryga et al. [44] reported fallapplied AA in Iowa soils with $\mathrm{pH}>7.5$ to have faster nitrification rates and more nitrate loss through denitrification during spring rainfall than AA applied in the spring. These results suggest that there was a significant amount of nitrate loss through denitrification in 2013 because of the relatively wet month of April $(194 \mathrm{~mm})$, which ultimately delayed planting to May.

A significant three-way interaction $(P \leq 0.05)$ between the timing of $\mathrm{N}$ application, source/placement, and the year was detected for corn grain yield at $168 \mathrm{~kg} \cdot \mathrm{N} \cdot \mathrm{ha}^{-1}$ (Table 3). In 2011, fall-applied deep UAN, surface NF, deep NF, and deep AA resulted in 3.6, 1.18, 2.41, and 2.70 Mg.ha ${ }^{-1}$ higher corn grain yield than an equivalent spring $\mathrm{N}$ application, respectively (Table 5 ). Since higher corn grain yields with fall $\mathrm{N}$ application were observed in 2011 for both 84 and $168 \mathrm{~kg} \cdot \mathrm{N} \cdot \mathrm{ha}^{-1}$, we can summarize that greater $\mathrm{N}$ loss was probably attributed to spring application, while NT/surface broadcast and ST/deep banded treatments were able to effectively minimize the potential for denitrification loss over the fall, winter, and early spring periods. Furthermore, corn grain yields in 2011 at $168 \mathrm{~kg} \cdot \mathrm{N} \cdot \mathrm{ha}^{-1}$ with spring $\mathrm{N}$ application of surface UAN and surface NF had at least a 1.75 and $1.02 \mathrm{Mg} \cdot \mathrm{ha}^{-1}$ higher corn grain yield than deep AA treatment, respectively. This was similar at $84 \mathrm{~kg} \cdot \mathrm{N} \cdot \mathrm{ha}^{-1}$, where surface UAN and surface NF in the spring had at least $0.98 \mathrm{Mg} \cdot \mathrm{ha}^{-1}$ higher corn grain yields in 2011 than deep UAN, NF, and AA treatments. This indicates that saturated soil conditions from more frequent precipitation events in the early growing season may have limited the growth of corn roots in the poorly drained soil so that roots were unable to absorb the deep banded fertilizer placement, but they were able to utilize the surface $\mathrm{N}$ fertilizer placement. The relatively low hydraulic conductivity of the claypan subsoil may have caused extended periods of soil saturation and negatively impacted corn grain yield with deep $\mathrm{N}$ placement. Another study at the same location concluded that surface applied UAN was relatively more effective for corn production with abundant early-season rainfall, which 
likely incorporated $\mathrm{N}$ from the fertilizer into the shallow soil layer and made $\mathrm{N}$ more available for plant uptake [17].

Similar to the results with an $\mathrm{N}$ rate of $84 \mathrm{~kg} \cdot \mathrm{ha}^{-1}$, corn grain yields were negatively affected by drought conditions in 2012 at $168 \mathrm{~kg} \cdot \mathrm{N} \cdot \mathrm{ha}^{-1}$ (Table 5). There were no significant differences among all sources/placements, timing, or $\mathrm{N}$ rates. Average grain yields for 2012 at 84 and $168 \mathrm{~kg} \cdot \mathrm{N} \cdot \mathrm{ha}^{-1}$ were 1.9 and $1.5 \mathrm{Mg} \cdot \mathrm{ha}^{-1}$, respectively.

The spring application of surface NF at $168 \mathrm{~kg} \cdot \mathrm{N} \cdot \mathrm{ha}^{-1}$ in 2013 had the highest corn grain yield among spring- or fallapplied $\mathrm{N}$ sources/placements. This result was likely due to the slow release rate of urea- $\mathrm{N}$ in NF because gaseous $\mathrm{N}$ loss may have been reduced by this $\mathrm{N}$ source prior to the period of substantial plant $\mathrm{N}$ uptake [33]. Although not significant, the surface NF treatment applied in the spring at $168 \mathrm{~kg} \cdot \mathrm{N} \cdot \mathrm{ha}^{-1}$ produced $0.67 \mathrm{Mg} \cdot \mathrm{ha}^{-1}$ higher grain yield compared to a fall application in 2013 (Table 5). This was potentially due to higher soil temperatures during 2013 and greater cumulative rainfall in May (Figure 1(c)). Relatively high rainfall in April 2013 delayed planting to May, thus potentially causing fall surface NF to be less effective, and has a greater risk of $\mathrm{N}$ loss before planting. This also suggests that spring-applied NF may have conserved N in the soil profile by delaying the conversion to nitrate until after the heavy rainfall period in May, regardless of placement. Shapiro et al. [8] found similar results where surface broadcast and subsurface banded UAN with NF produced 0.21 to $0.59 \mathrm{Mg} \cdot \mathrm{ha}^{-1}$ higher grain yields than surface broadcast UAN without NF on a loamy sand soil at a site in Nebraska. Although the study did not assess different $\mathrm{N}$ timings, the research was still similar because cumulative rainfall was greater in May $(252 \mathrm{~mm})$ at this particular Nebraska location compared to other sites in the experiment.

In 2013, deep AA yielded $1.7 \mathrm{Mg} \cdot \mathrm{ha}^{-1}$ more grain when spring-applied compared to when it was fall-applied at $168 \mathrm{~kg} \cdot \mathrm{N} \cdot \mathrm{ha}^{-1}$. This suggests that $\mathrm{N}$ loss may have occurred through denitrification or volatilization over the fall, winter, and early spring periods. This is similar to a 3-year study done in Minnesota that observed an average increase of $0.8 \mathrm{Mg} \cdot \mathrm{ha}^{-1}$ in corn grain yield with AA applied in the spring compared to fall application [7]. Furthermore, research done on a poorly drained claypan soil reported a $2.0 \mathrm{Mg} \cdot \mathrm{ha}^{-1}$ reduction in corn grain yield with fall-applied AA at $140 \mathrm{~kg} \cdot \mathrm{N} \cdot \mathrm{ha}^{-1}$ compared to a spring application [6]. Similarly, Nash et al. [6] found fall application of ammonium nitrate produced less corn grain yield than spring $\mathrm{N}$ application which may have been due to less soil retention.

Spring and fall application of NT/surface broadcast and ST/deep banded sources/placements was similar in 2013 at $168 \mathrm{~kg} \cdot \mathrm{N} \cdot \mathrm{ha}^{-1}$ except for deep AA (Table 5). The inconsistent corn grain yields among the three years in this experiment can be attributed to the variations in precipitation that occurred, especially after the spring $\mathrm{N}$ application. Rainfall was relatively more frequent after spring $\mathrm{N}$ application in 2011 and may have prolonged saturated soil conditions and caused ST/deep banded treatments to be ineffective because of spatial and temporal $\mathrm{N}$ shortages in the root zone. Although deep banding placement may conserve $\mathrm{N}$, there may be spatial and temporal $\mathrm{N}$ shortages because $\mathrm{N}$ may be spatially separated from plant roots throughout significant parts of the growing season [8]. Furthermore, cumulative precipitation was relatively low between fall $\mathrm{N}$ application (November 2010) and planting, suggesting minimal $\mathrm{N}$ loss may have occurred during the fall, winter, and early spring months, while more $\mathrm{N}$ was potentially lost in the months following the spring $\mathrm{N}$ application (Figure 1). Fall $\mathrm{N}$ application may have retained more $\mathrm{NO}_{3}^{-}$and $\mathrm{NH}_{4}{ }^{+}$in the soil by spring in 2011, which means it was more readily available for plant uptake during the early growing season [12]. Vetsch and Randall [7] observed greater soil $\mathrm{NO}_{3}-\mathrm{N}$ concentrations with fall-applied $\mathrm{N}$ than spring-applied $\mathrm{N}$, indicating substantial nitrification of fall-applied $\mathrm{N}$ occurred by the early growth stages in the spring.

High corn grain yields in 2013 with spring $\mathrm{N}$ application of deep AA may have been due to an increase in microbial activity from warmer soil conditions in May. There was $27 \mathrm{~mm}$ of precipitation that occurred on the same day for fall $\mathrm{N}$ application (11 November 2012), which may have caused deep AA to lose a significant amount of $\mathrm{N}$ due to physical disruption and improper sealing of the injection knives that may have created zones to allow for ammonia movement to reach the soil surface [45] or distribution of $\mathrm{N}$ with the application knives may have allowed greater $\mathrm{N}$ loss [34]. Implementing different $\mathrm{N}$ management practices is a good option for farmers to increase corn grain yield. One study combined NT with subsurface UAN or AA placement and observed higher corn grain yield compared to utilizing a conventional tillage system with the same $\mathrm{N}$ application [46]. This likely resulted from decreased ammonia volatilization and immobilization commonly associated with surface $\mathrm{N}$ application on high-residue soil surfaces.

3.6. Grain Moisture and Quality. Corn grain moisture concentration had a significant interaction between $\mathrm{N}$ source/placement and $\mathrm{N}$ timing $(P \leq 0.05)$ when averaged across years at $84 \mathrm{~kg} \cdot \mathrm{N} \cdot \mathrm{ha}^{-1}$ (Table 3 ). Deep UAN had higher corn grain moisture with spring $\mathrm{N}$ application than fall $\mathrm{N}$ application at $84 \mathrm{~kg} \cdot \mathrm{N} \cdot \mathrm{ha}^{-1}$ (Table 6). Conversely, surface NF and deep AA had 17 and $16 \mathrm{~g} \cdot \mathrm{kg}^{-1}$ higher corn grain moisture with fall $\mathrm{N}$ application compared to spring $\mathrm{N}$ application at $84 \mathrm{~kg} \cdot \mathrm{N} \cdot \mathrm{ha}^{-1}$, respectively. Deep UAN in the spring had corn grain moisture of $223 \mathrm{~g} \cdot \mathrm{kg}^{-1}$ which was $25 \%$ more than the deep UAN treatment in fall at $84 \mathrm{~g} \cdot \mathrm{kg}^{-1}$ (Table 6). Overall, grain was drier with surface placement of $\mathrm{N}$ compared to deep placement.

At $168 \mathrm{~kg} \cdot \mathrm{N} \cdot \mathrm{ha}^{-1}$, corn grain moisture was different among $\mathrm{N}$ sources/placements $(P \leq 0.05)$ regardless of $\mathrm{N}$ timing and year (Tables 3 and 5). Deep NF $\left(207 \mathrm{~g} \cdot \mathrm{kg}^{-1}\right)$ and deep AA $\left(215 \mathrm{~g} \cdot \mathrm{kg}^{-1}\right)$ at $168 \mathrm{~kg} \cdot \mathrm{N} \cdot \mathrm{ha}^{-1}$ had at least $17 \mathrm{~g} \cdot \mathrm{kg}^{-1}$ greater moisture concentration than all other $\mathrm{N}$ sources/ placements (Table 5). Surface NF at $168 \mathrm{~kg} \cdot \mathrm{N} \cdot \mathrm{ha}^{-1}$ resulted in the lowest grain moisture content $\left(175 \mathrm{~g} \cdot \mathrm{kg}^{-1}\right)$ and was not significantly different from surface UAN $\left(181 \mathrm{~g} \cdot \mathrm{kg}^{-1}\right)$ or deep UAN $\left(190 \mathrm{~g} \cdot \mathrm{kg}^{-1}\right)$. Corn grain moisture content is affected by several factors including but not limited to $\mathrm{N}$ fertilizer type, corn hybrid, tillage system, or climate $[40,47]$. All of these factors influence the rate of corn maturation, in which 
TABLE 6: Corn grain moisture, test weight, protein, and oil concentration response to nitrogen sources $/$ placements at $84 \mathrm{~kg} \cdot \mathrm{N} \cdot \mathrm{ha}{ }^{-1}$. Data were combined over factors or years in the absence of a significant interaction. Surface treatments were no-tillage with surface applied nitrogen and deep treatments were strip tillage with deep banded nitrogen application.

\begin{tabular}{|c|c|c|c|c|c|c|c|}
\hline \multirow{2}{*}{$\mathrm{N}$ source placement } & \multicolumn{2}{|c|}{ Moisture } & \multirow{2}{*}{ Test weight } & \multicolumn{3}{|c|}{ Protein } & \multirow{2}{*}{ Oil } \\
\hline & $\mathrm{F}$ & $S$ & & 2011 & 2012 & 2013 & \\
\hline & \multicolumn{2}{|c|}{$\mathrm{g} \cdot \mathrm{kg}^{-1}$} & $\mathrm{~kg} \cdot \mathrm{hL}^{-1}$ & & $\mathrm{~g} \cdot \mathrm{kg}^{-1}$ & & \\
\hline Surface $\mathrm{UAN}^{\dagger}$ & 187 & 194 & 55.7 & 72.9 & 100.9 & 67.9 & 34 \\
\hline Deep UAN & 178 & 223 & 53.3 & 87.2 & 103 & 72 & 32.9 \\
\hline Surface NF & 190 & 173 & 56.1 & 72 & 101.5 & 70 & 33.9 \\
\hline Deep NF & 201 & 224 & 54.9 & 84.6 & 102.3 & 66.1 & 33.2 \\
\hline Deep AA & 217 & 201 & 55.3 & 88.1 & 102.3 & 70 & 33.4 \\
\hline $\operatorname{LSD}(P \leq 0.05)^{\ddagger}$ & \multicolumn{2}{|c|}{42} & NS & & 2.1 & & NS \\
\hline
\end{tabular}

${ }^{\dagger}$ Abbreviations: AA, anhydrous ammonia; F, fall; NF, Nitamin Nfusion; NS, not significant; S, spring; UAN, 32\% urea ammonium nitrate. ${ }^{*}$ Fisher's protected least significant difference at $P \leq 0.05$.

TABLE 7: Fall and spring chlorophyll meter readings, moisture, test weight, and oil concentration response to nitrogen sources at $168 \mathrm{~kg} \cdot \mathrm{N} \cdot \mathrm{ha}^{-1}$. Data were combined over years and N sources/placements.

\begin{tabular}{lccc}
\hline $\mathrm{N}$ timing & Chlorophyll meter readings & Moisture & Test weight \\
\hline & SPAD units & $\mathrm{g} \cdot \mathrm{kg}^{-1}$ & $\mathrm{~kg} \cdot \mathrm{hL}^{-1}$ \\
Fall & 52.6 & 191 & 68.0 \\
Spring & 51.9 & 196 & 69.6 \\
LSD $(P \leq 0.05)^{\ddagger}$ & $\mathrm{NS}^{\dagger}$ & $\mathrm{NS}$ & 32.7 \\
\hline
\end{tabular}

${ }^{\dagger} \mathrm{NS}$, not significant. ${ }^{*}$ Fisher's protected least significant difference at $P \leq 0.05$.

a slower rate generally results in relatively higher grain moisture content [48].

Protein concentration in corn grain had an interaction between $\mathrm{N}$ sources/placements and year $(P \leq 0.05)$ at $84 \mathrm{~kg} \cdot \mathrm{N} \cdot \mathrm{ha}^{-1}$ (Table 3). Deep NF in 2013 had the lowest grain protein concentration $\left(66.1 \mathrm{~g} \cdot \mathrm{kg}^{-1}\right)$ than all $\mathrm{N}$ source placements in the three years (Table 6). Overall, grain protein concentration for $\mathrm{N}$ sources/placements in 2012 ranged from 100.9 to $103.0 \mathrm{~g} \cdot \mathrm{kg}^{-1}$ and was at least $12.8 \mathrm{~g} \cdot \mathrm{kg}^{-1}$ higher than treatments in 2011 and 2013. The higher grain protein content in 2012 was probably due to drought conditions that strongly restricted plant growth.

Protein concentration in corn grain also had a significant interaction between $\mathrm{N}$ sources/placements and year $(P<0.05)$ at $168 \mathrm{~kg} \cdot \mathrm{N} \cdot \mathrm{ha}^{-1}$ (Tables 3 and 5). Protein concentration in corn grain was highest for all $\mathrm{N}$ sources/ placements (103.8 to $104.8 \mathrm{~g} \cdot \mathrm{kg}^{-1}$ ) in 2012 (Table 5). Surface UAN in 2013 had a lower grain protein concentration $\left(75.9 \mathrm{~g} \cdot \mathrm{kg}^{-1}\right)$ than all $\mathrm{N}$ sources/placements in the three years of this research, but it was similar to deep AA in 2013 and surface UAN $\left(77.8 \mathrm{~g} \cdot \mathrm{kg}^{-1}\right)$ or surface NF $\left(77.5 \mathrm{~g} \cdot \mathrm{kg}^{-1}\right)$ in 2011 (Table 5). Generally, the results of grain protein concentration corresponded with SPAD meter leaf readings since both SPAD meter readings and grain protein concentration had similar patterns in 2011,2012, and 2013. Corn grain protein concentration is also potentially an indicator for $\mathrm{N}$ uptake and removal by the plant since protein concentration is usually calculated from grain $\mathrm{N}$ concentration.

Corn grain oil concentrations at $84 \mathrm{~kg} \cdot \mathrm{N} \cdot \mathrm{ha}^{-1}$ were combined over years since there was no significant interaction observed (Table 3). Surface UAN had the highest grain oil concentration $\left(34.0 \mathrm{~g} \cdot \mathrm{kg}^{-1}\right)$, but it was similar to the other $\mathrm{N}$ source/placement treatments (Table 6). Corn grain oil concentrations were different among $\mathrm{N}$ source placements at
$168 \mathrm{~kg} \cdot \mathrm{N} \cdot \mathrm{ha}^{-1}$ (Tables 3 and 5). Grain oil concentration was 1.7 and $2 \mathrm{~g} \cdot \mathrm{kg}^{-1}$ higher with deep AA than surface NF and deep UAN, respectively (Table 5). Deep UAN at $168 \mathrm{~kg} \cdot \mathrm{N} \cdot \mathrm{ha}^{-1}$ had 1.5 and $1.6 \mathrm{~g} \cdot \mathrm{kg}^{-1}$ lower grain oil concentration than surface UAN and deep NF. Combining N source placements across the three years at $168 \mathrm{~kg} \cdot \mathrm{N} \cdot \mathrm{ha}^{-1}$ indicated higher grain oil concentration with the fall application $\left(32.7 \mathrm{~g} \cdot \mathrm{kg}^{-1}\right)$ compared to a spring application $\left(31.7 \mathrm{~g} \cdot \mathrm{kg}^{-1}\right)$ (Table 7).

\section{Conclusions}

Utilizing different $\mathrm{N}$ management strategies for corn production can be challenging due to unpredictable year-toyear climate conditions. On claypan soils, corn production is even more difficult to manage due to these soils' relatively poor drainage characteristics, which contribute to a higher potential for $\mathrm{N}$ loss and subsequently lower crop yields as well as the propensity to drought. Although the data were variable across $\mathrm{N}$ timings, $\mathrm{N}$ sources/placements, and year, this range of climatic conditions can be expected in the US corn belt region. Over this three-year study, the application of strip-till deep banded UAN in the fall before the 2011 growing season resulted in the highest corn grain yield (8.12 to $9.12 \mathrm{Mg} \cdot \mathrm{ha}^{-1}$ ) at both 84 and $168 \mathrm{~kg} \cdot \mathrm{N} \cdot \mathrm{ha}^{-1}$. This indicates greater $\mathrm{N}$ loss was attributed to spring application in 2011 due to cooler soil conditions and extended periods of soil saturation early in the growing season. Thus, climate conditions may have caused spatial and temporal $\mathrm{N}$ shortages when $\mathrm{N}$ was deep banded as UAN or AA fertilizer because it may be separated from plant roots or may not be converted into $\mathrm{NO}_{3}-\mathrm{N}$ at the appropriate growth stage. Spring $\mathrm{N}$ application showed an increase in corn grain yield in 2013 among treatments compared to 2011, which may have been due to warmer and wetter soil conditions in May. This study 
suggests that ST with deep banding UAN or AA does not always produce higher corn grain yield during drought years and might be more at risk for greater $\mathrm{N}$ loss under certain climatic conditions. On a poorly drained claypan soil, farmers may need to consider fall $\mathrm{N}$ applications since corn grain yield was generally greater than or equal to springapplied treatments at both rates.

\section{Data Availability}

The data used to support the findings of this study are available from the corresponding author upon request.

\section{Conflicts of Interest}

The authors declare that they have no conflicts of interest regarding the publication of this paper.

\section{Acknowledgments}

The authors would like to thank the Missouri Agriculture Experiment Station for their financial support of the project.

\section{References}

[1] NRCS, Land Resource Regions and Major Land Resource Areas of the United States, the Caribbean, and the Pacific Basin, U.S. Government Printing Office, Washington, DC, USA, 2006.

[2] W. K. Jung, N. R. Kitchen, K. A. Sudduth, and S. H. Anderson, "Spatial characteristics of claypan soil properties in an agricultural field," Soil Science Society of America Journal, vol. 70, no. 4, pp. 1387-1397, 2006.

[3] K. A. Nelson, S. M. Paniagua, and P. P. Motavalli, "Effect of polymer coated urea, irrigation, and drainage on nitrogen utilization and yield of corn in a claypan soil," Agronomy Journal, vol. 101, no. 3, pp. 681-687, 2009.

[4] T. Bruulsema, J. Lemunyon, and B. Herz, "Know your fertilizer rights," Crops and Soils, vol. 42, no. 2, pp. 13-18, 2009.

[5] J. Sawyer, E. Nafziger, G. Randall, L. Bundy, G. Rehm, and B. Joern, Concepts and Rationale for Regional Nitrogen Rate Guidelines for Corn, Iowa State University Extension and Outreach Publications, Ames, IA, USA, 2006.

[6] P. R. Nash, K. A. Nelson, and P. P. Motavalli, "Corn yield response to timing of strip-tillage and nitrogen source applications," Agronomy Journal, vol. 105, no. 3, pp. 623-630, 2013.

[7] J. A. Vetsch and G. W. Randall, "Corn production as affected by nitrogen application timing and tillage," Agronomy Journal, vol. 96, no. 2, pp. 502-509, 2004.

[8] C. Shapiro, A. Attia, S. Ulloa, and M. Mainz, "Use of five nitrogen source and placement systems for improved nitrogen management of irrigated corn," Soil Science Society of America Journal, vol. 80, no. 6, pp. 1663-1674, 2016.

[9] G. W. Randall and M. A. Schmitt, "Advisability of fall-applying nitrogen," in Proceedings of the 1998 Wisconsin Fertilizer, Aglime, and Pest Management Conference, UW College of Agricultural \& Life Sciences, Madison, WI, USA, January 1998.

[10] D. L. Dinnes, D. L. Karlen, D. B. Jaynes et al., "Nitrogen management strategies to reduce nitrate leaching in tiledrained Midwestern soils," Agronomy Journal, vol. 94, no. 1, pp. 153-171, 2002.

[11] G. W. Randall and D. J. Mulla, "Nitrate nitrogen in surface waters as influenced by climatic conditions and agricultural practices," Journal of Environment Quality, vol. 30, no. 2, pp. 337-344, 2001.

[12] G. W. Randall, J. A. Vetsch, and J. R. Huffman, "Corn production on a subsurface-drained mollisol as affected by time of nitrogen application and nitrapyrin," Agronomy Journal, vol. 95, no. 5, pp. 1213-1219, 2003.

[13] J. D. Stamper, Evaluation of Method of Placement, Timing, and Rate of Application for Anhydrous Ammonia in No-Till Corn Production, Kansas State University, Manhattan, KS, USA, 2009.

[14] L. Bundy, "Review: timing nitrogen applications to maximize fertilizer efficiency and crop response in conventional corn production," Journal of Fertilizer Issues, vol. 3, no. 3, pp. 99-106, 1986.

[15] T. W. Steusloff, K. A. Nelson, P. P. Motavalli, and G. Singh, "Fertilizer placement affects corn and nitrogen use efficiency in a claypan soil," Agronomy Journal, vol. 111, no. 4, 2019.

[16] A. D. Halvorson, A. R. Mosier, C. A. Reule, and W. C. Bausch, "Nitrogen and tillage effects on irrigated continuous corn yields," Agronomy Journal, vol. 98, no. 1, pp. 63-71, 2006.

[17] F. E. Johnson II, K. A. Nelson, and P. P. Motavalli, "Urea fertilizer placement impacts on corn growth and nitrogen utilization in a poorly-drained claypan soil," Journal of Agricultural Science, vol. 9, no. 1, pp. 28-40, 2016.

[18] P. M. Nkebiwe, M. Weinmann, A. Bar-Tal, and T. Müller, "Fertilizer placement to improve crop nutrient acquisition and yield: a review and meta-analysis," Field Crops Research, vol. 196, pp. 389-401, 2016.

[19] K. Paustian, B. Babcock, J. Hatfield et al., "Climate change and greenhouse gas mitigation: challenges and opportunities for agriculture," CAST Task Force Report, Council for Agricultural Science and Technology, Ames, IA, USA, 2004.

[20] W. E. Riedell, D. L. Beck, and T. E. Schumacher, "Corn response to fertilizer placement treatments in an irrigated no-till system," Agronomy Journal, vol. 92, no. 2, pp. 316-320, 2000.

[21] G. W. Randall and P. R. Hill, "Fall strip-tillage systems," in Conservation Tillage Systems and Management, R. C. Reeder, Ed., pp. 193-199, Iowa State University, Ames, IA, USA, 2000.

[22] B. B. Mehdi, C. A. Madramootoo, and G. R. Mehuys, "Yield and nitrogen content of corn under different tillage practices," Agronomy Journal, vol. 91, no. 4, pp. 631-636, 1999.

[23] B. J. Hendrix, B. G. Young, and S.-K. Chong, "Weed management in strip tillage corn," Agronomy Journal, vol. 96, no. 1, pp. 229-235, 2004.

[24] A. D. Halvorson and S. J. Del Grosso, "Nitrogen source and placement effects on soil nitrous oxide emissions from no-till corn," Journal of Environment Quality, vol. 41, no. 5, pp. 1349-1360, 2012.

[25] P. Rochette, D. A. Angers, M. H. Chantigny et al., "Ammonia volatilization and nitrogen retention: how deep to incorporate urea?," Journal of Environment Quality, vol. 42, no. 6, pp. 1635-1642, 2013.

[26] T. S. Steusloff, K. A. Nelson, P. P. Motavalli, and G. Singh, "Urea nitrapyrin placement effects on soil nitrous oxide emissions in claypan soil," Journal of Environmental Quality, 2019.

[27] S. Cahill, D. Osmond, R. Weisz, and R. Heiniger, "Evaluation of alternative nitrogen fertilizers for corn and winter wheat production," Agronomy Journal, vol. 102, no. 4, pp. 1226-1236, 2010.

[28] A. D. Halvorson and M. E. Bartolo, "Nitrogen source and rate effects on irrigated corn yields and nitrogen-use efficiency," Agronomy Journal, vol. 106, no. 2, pp. 681-693, 2014.

[29] A. J. Noellsch, P. P. Motavalli, K. A. Nelson, and N. R. Kitchen, "Corn response to conventional and slowrelease nitrogen fertilizers across a claypan landscape," Agronomy Journal, vol. 101, no. 3, pp. 607-614, 2009. 
[30] R. T. Venterea, M. Bijesh, and M. S. Dolan, "Fertilizer source and tillage effects on yield-scaled nitrous oxide emissions in a corn cropping system," Journal of Environment Quality, vol. 40, no. 5, pp. 1521-1531, 2011.

[31] K. R. Sistani, M. Jn-Baptiste, and J. R. Simmons, "Corn response to enhanced-efficiency nitrogen fertilizers and poultry litter," Agronomy Journal, vol. 106, no. 2, pp. 761-770, 2014.

[32] J. Phillips, S. Wertz, and A. Cothran, "High nitrogen liquid fertilizer," Google Patents US 2006196241-A1, 2006.

[33] P. R. Nash, P. P. Motavalli, and K. A. Nelson, "Nitrous oxide emissions from claypan soils due to nitrogen fertilizer source and tillage/fertilizer placement practices," Soil Science Society of America Journal, vol. 76, no. 3, pp. 983-993, 2012.

[34] P. Lanpher, "Conservation farming strip till nitrogen applicator," Google Patents US 6382114B1, 2002.

[35] L. J. Abendroth, R. W. Elmore, M. J. Boyer, and S. K. Marlay, Corn Growth and Development, Iowa State University Extension, Ames, IA, USA, 2011.

[36] K. A. Nelson, "Pronitridine nitrification inhibitor with urea ammonium nitrate for corn," Journal of Agricultural Science, vol. 10, no. 6, pp. 16-25, 2018.

[37] T. W. Andraski and L. G. Bundy, "Corn residue and nitrogen source effects on nitrogen availability in no-till corn," Agronomy Journal, vol. 100, no. 5, pp. 1274-1279, 2008.

[38] S. Agehara and D. D. Warncke, "Soil moisture and temperature effects on nitrogen release from organic nitrogen sources," Soil Science Society of America Journal, vol. 69, no. 6, pp. 1844-1855, 2005.

[39] D. R. Griffith, J. V. Mannering, H. M. Galloway, S. D. Parsons, and C. B. Richey, "Effect of eight tillage-planting systems on soil temperature, percent stand, plant growth, and yield of corn on five Indiana soils," Agronomy Journal, vol. 65, no. 2, pp. 321-326, 1973.

[40] J. A. Vetsch and G. W. Randall, "Corn production as affected by tillage system and starter fertilizer," Agronomy Journal, vol. 94 , no. 3, pp. 532-540, 2002.

[41] C. F. Drury, W. D. Reynolds, C. S. Tan, T. W. Welacky, W. Calder, and N. B. McLaughlin, "Emissions of nitrous oxide and carbon dioxide," Soil Science Society of America Journal, vol. 70, no. 2, pp. 570-581, 2006.

[42] C. A. Grant, D. A. Derksen, D. McLaren, and R. B. Irvine, "Nitrogen fertilizer and urease inhibitor effects on canola emergence and yield in a one-pass seeding and fertilizing system," Agronomy Journal, vol. 102, no. 3, pp. 875-884, 2010.

[43] A. S. Lithourgidis, C. A. Tsatsarelis, and K. V. Dhima, "Tillage effects on corn emergence, silage yield, and labor and fuel inputs in double cropping with wheat," Crop Science, vol. 45, no. 6, pp. 2523-2528, 2005.

[44] P. M. Kyveryga, A. M. Blackmer, J. W. Ellsworth, and R. Isla, "Soil pH effects on nitrification of fall-applied anhydrous ammonia," Soil Science Society of America Journal, vol. 68, no. 2, pp. 545-551, 2004.

[45] C. J. Overdahl and G. W. Rehm, Using Anhydrous Ammonia in Minnesota, University of Minnesota Extension Service, St. Paul, MN, USA, 1990.

[46] D. B. Mengel, D. W. Nelson, and D. M. Huber, "Placement of nitrogen fertilizers for no-till and conventional till corn," Agronomy Journal, vol. 74, no. 3, pp. 515-518, 1982.

[47] R. Wolkowski, "Row-placed fertilizer for maize grown with an in-row crop residue management system in Southern Wisconsin," Soil and Tillage Research, vol. 54, no. 1-2, pp. 55-62, 2000.

[48] D. B. Beegle, G. W. Roth, and D. D. Lingenfelter, Starter Fertilizer, Agronomy Facts 51, Penn State College of Agricultural Sciences. The Pennsylvania State University, University Park, State College, PA, USA, 2003. 


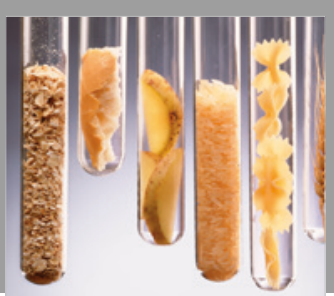

International Journal of Food Science

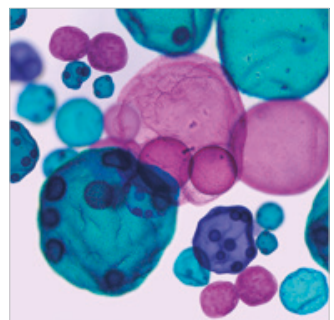

International Journal of Microbiology
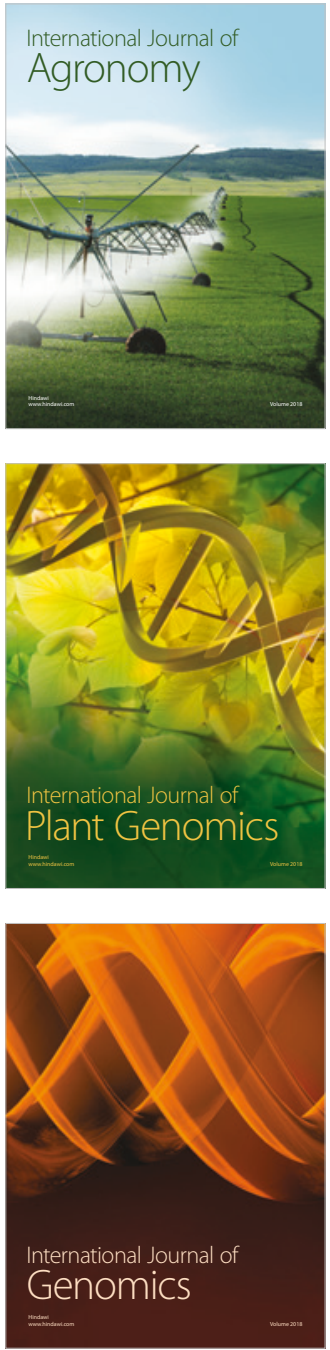

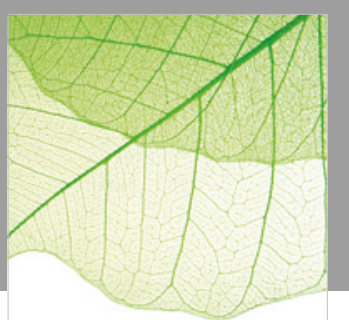

Journal of Botany
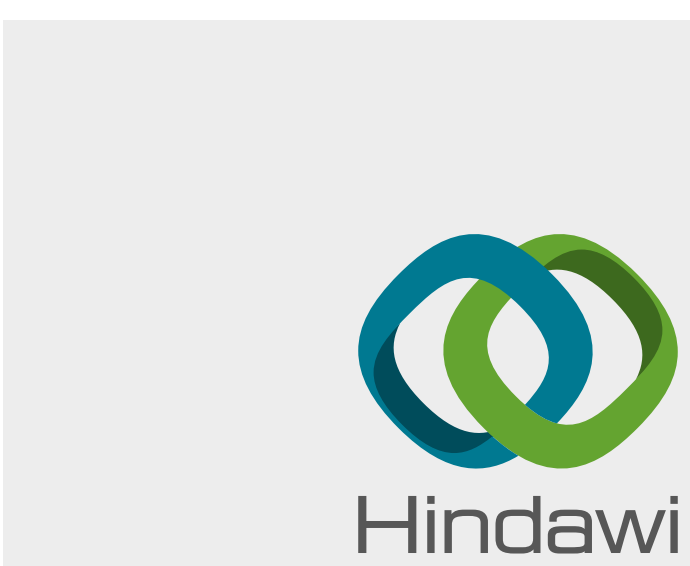

Submit your manuscripts at

www.hindawi.com
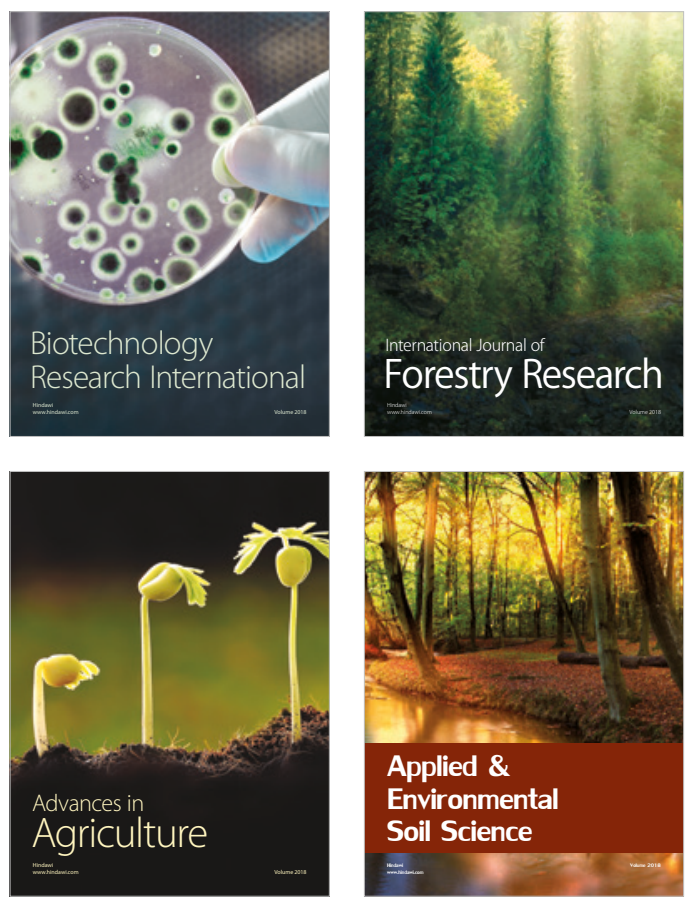

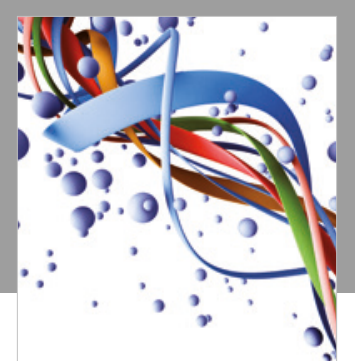

Scientifica

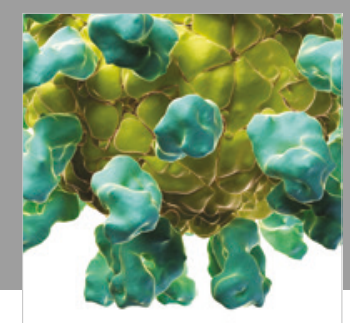

Veterinary Medicine International

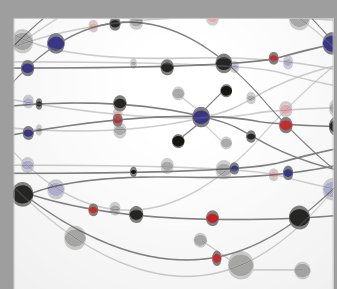

The Scientific World Journal
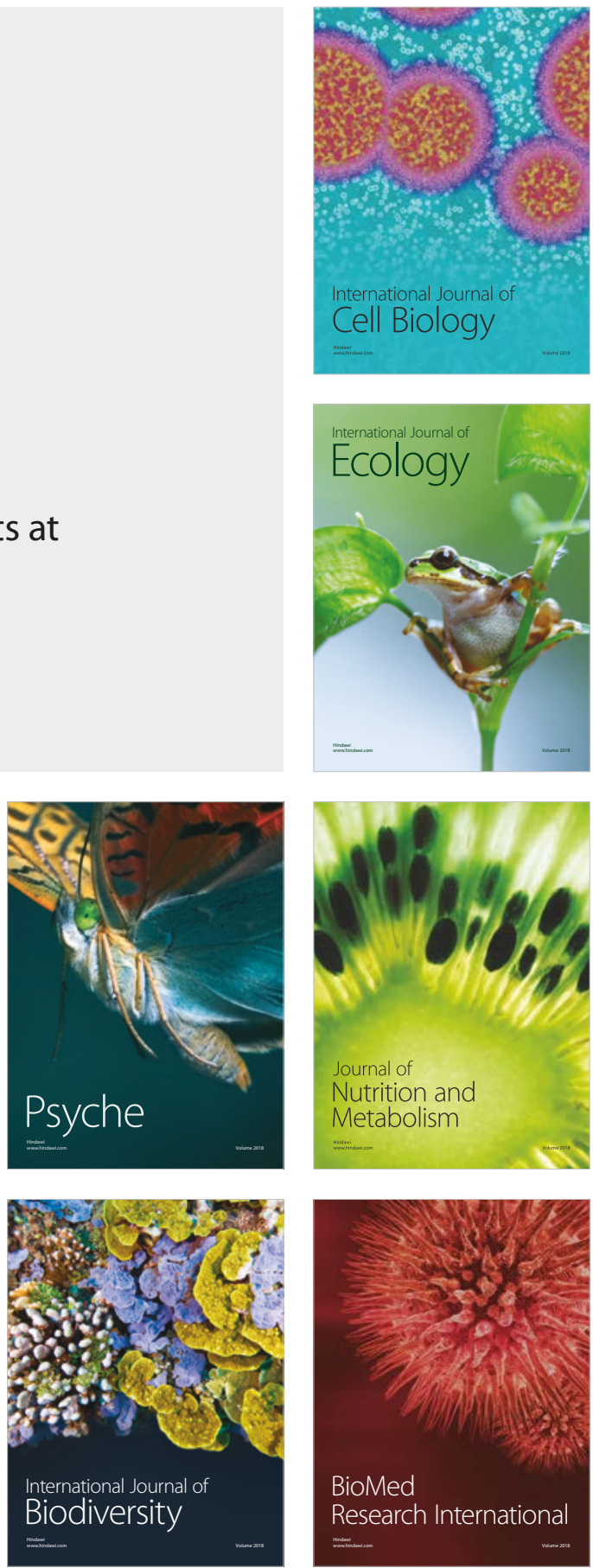